Purdue University Purdue e-Pubs

$10-1-2005$

\title{
Laser bending for adjusting curvatures of hard disk suspensions
}

Xi R. Zhang

Purdue University

Xianfan Xu

Birck Nanotechnology Center, School of Materials Engineering, Purdue University, xxu@purdue.edu

Follow this and additional works at: http://docs.lib.purdue.edu/nanodocs

Zhang, Xi R. and Xu, Xianfan, "Laser bending for adjusting curvatures of hard disk suspensions" (2005). Other Nanotechnology Publications. Paper 53.

http://docs.lib.purdue.edu/nanodocs/53

This document has been made available through Purdue e-Pubs, a service of the Purdue University Libraries. Please contact epubs@purdue.edu for additional information. 


\section{Laser bending for adjusting curvatures of hard disk suspensions}

\begin{abstract}
The purpose of this work is to use 3D finite element analyses to compute bending of a hard disk suspension using a laser. The pitch and roll of the suspension can be precisely adjusted by producing a controlled amount of residual strain using the laser as a heat source. In the computational model, an uncoupled thermo-mechanical analysis is applied to calculate the laser induced thermal loading and mechanical deformation. The relation between suspension bending and laser parameters is studied based on extensive simulations. Bending resolution as high as $0.01^{\circ}$ can be achieved. In addition, a data table is established where laser parameters, such as laser power and laser scan length can be found to correct a given pitch and roll of a hard disk suspension. Effects of uncertainties, such as sample thickness and material yield strength are also studied.
\end{abstract}

\section{Introduction}

Suspensions are the mechanical support and dynamic spring that holds the magnetic recording heads over the surface of a hard disk. It is the suspension assembly that allows the recording head to "fly" over the disk surface at a height on the order of nanometers. These suspensions have to be relatively stiff in lateral translation, but flexible in pitch and roll. A schematic of the hard disk suspension is shown in Fig. 1. The dark part is the main part of the suspension and it is made of stainless steel, and is welded onto the thick base underneath it. Some

X. R. Zhang $\cdot$ X. Xu ( $($ )

School of Mechanical Engineering, Purdue University,

West Lafayette, Indiana 47907, USA

E-mail: xxu@ecn.purdue.edu

Tel.: 1-765-4945639

Fax: + 1-765-4940539 imperfections of the suspension can occur as a result of manufacturing processes, mainly the pitch and roll angles. The pitch angle is defined as the angle rotating along the line $\mathrm{A}-\mathrm{A}$, and the roll angle as the angle rotating along the line B-B. A large amount of experimental and numerical work has been conducted to study suspension modeling and optimization (Wilson and Bogy 1994; Takahashi et al. 1998; Bogy and Zeng 1999; Frank et al. 2000; Kilian et al. 2003; Weissner et al. 2003). However, little work has been done on high precision curvature or pitch/roll adjustment.

It is difficult to use traditional methods to produce high precision deformation to hard drive suspensions. On the other hand, laser-based microfabrication is noncontact, flexible and cost effective. The laser spot size can be reduced to the order of micrometers using optical lenses. Therefore, the heat-affected zone can be very small. Very often, laser-based microfabrication is the only technique capable to achieve high precision. For instance, laser was used to adjust the suspension preload (gram load) on the slider to change the flying height (Singh et al. 2001). Recently, high precision laser bending for the hard drive read/write slider was demonstrated experimentally (Chen et al. 1998; Zhang and Xu 2003). A bending precision better than $1 \mu \mathrm{rad}$ was achieved.

Laser bending or laser forming is a technique of using the energy from a laser beam to modify the curvature of sheet metals or hard materials. Most laser bending processes involve the temperature gradient mechanism (TGM) (Chen et al. 1998; Zhang and Xu 2003; Geiger and Vollertsen 1993). When the laser beam irradiates the specimen surface, it produces a sharp temperature gradient in the thickness direction, causing the upper layer of the heated material to expand more than the lower layers. This non-uniform thermal expansion causes the target to bend away from the laser beam. In the meantime, compressive stress and strain are produced by the bulk constraint of the surrounding materials. Because of the high temperature achieved, plastic deformations occur. During cooling, heat flows into the adjacent area and the stress changes from compressive to tensile due to 


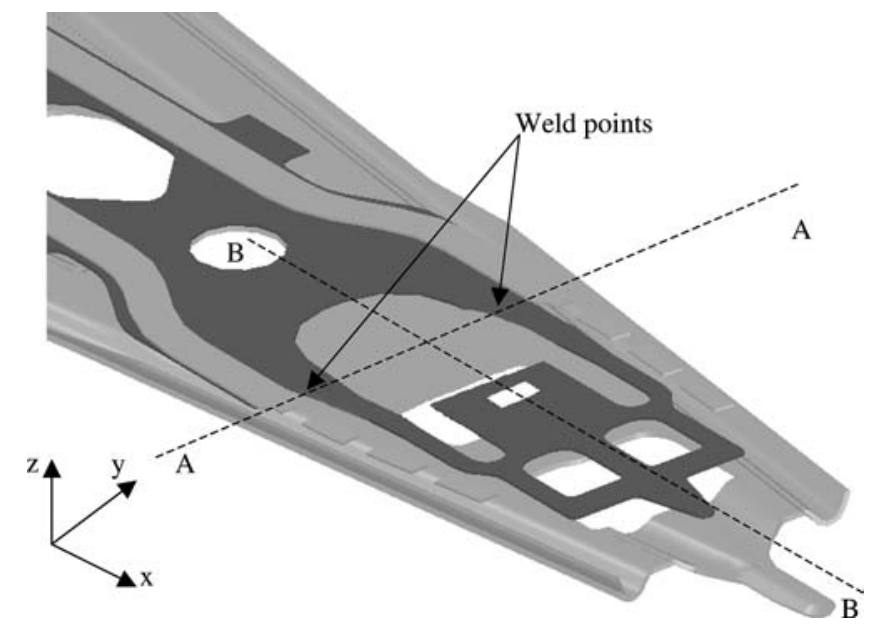

Fig. 1 AutoCAD drawing of a hard dist suspension

thermal shrinkage. However, the compressive strain generated during heating is not completely cancelled. Therefore, the residual strain in the laser-irradiated area is compressive after the target cools, causing a permanent bending deformation toward the laser beam. This theory of laser bending has been confirmed by a number of studies by comparing experimental data with results of finite element calculations (Chen et al. 1998; Zhang and $\mathrm{Xu} 2003$ ).

This paper presents a 3D finite element calculation of laser bending of suspensions. The difference between this work and previous works (Chen et al. 1998; Zhang and $\mathrm{Xu} 2003$ ) is that a much more complex geometry, i.e., the geometry of a suspension, is modeled to demonstrate the potential of using the laser bending technique in high precision curvature adjustment of a suspension. An uncoupled thermo-mechanical analysis is applied to calculate the laser induced thermal loading and mechanical deformation. The relation between suspension bending and laser parameters is studied based on extensive simulations. It is shown that undesired pitch and roll of a suspension can be corrected by choosing appropriate laser parameters.

\section{Numerical modeling}

In this paper, laser bending induced by the temperature gradient mechanism is calculated using 3D finite element models. A thermal analysis and a stress analysis are conducted. The two analyses are treated as uncoupled since the heat dissipation due to deformation is negligible compared with the heat provided by the lasers. In an uncoupled thermo-mechanical model, a transient temperature field is obtained first in the thermal analysis, and is then used as a thermal loading in the subsequent stress analysis to obtain transient stress, strain, and displacement distributions. The thermal analysis is based on solving the $3 \mathrm{D}$ heat conduction equation. The initial condition is that the whole specimen is at the room temperature $(300 \mathrm{~K})$. The $\mathrm{CW}$ laser flux is handled as a volumetric heat source decreasing exponentially from the target surface. The wavelength of the $\mathrm{CW}$ laser is $1.064 \mu \mathrm{m}$ and the optical absorption depth of stainless steel at this wavelength is $2 \times 10^{-8} \mathrm{~m}$ (Zhang and $\mathrm{Xu}$ 2003). Using the transient temperature data obtained from the thermal analysis as thermal loading, the transient stress, strain, and displacement distributions are obtained by solving the quasi-static force equilibrium equations. Details of thermal and stress analyses have been discussed elsewhere (Zhang and Xu 2003; Zhang et al. 2002).

The material is assumed to be linearly elastic-perfectly plastic. The Von Mises yield criterion is used to model the onset of plasticity. Sensitivity of simulation results to the plasticity assumption has been studied (Chen et al. 1999). Creep is neglected due to the short laser heating duration. Material properties of stainless steel 304 (which is the typical suspension material) including thermal conductivity, thermal expansion coefficient, density, yield stress, and Young's modulus are considered as temperature dependent (Maykuth 1980). Poisson's ratio is also considered as temperature dependent (Takeuti et al. 1979).

The computational domain of the 3D finite element analyses of laser bending of suspension is shown in Fig. 2. The thickness of the suspension is $20 \mu \mathrm{m}$. The total length from the end of the arm to the tip is $4.06 \mathrm{~mm}$ and the total width of the suspension is $1.68 \mathrm{~mm}$. The suspension is modeled as a cantilever, which is clamped at the left end. The two $600 \mu \mathrm{m}$ long regions (circled in Fig. 2) on the suspension arms are fine mesh regions, which are affected by laser irradiation. The maximum scan length is $200 \mu \mathrm{m}$ and the $600 \mu \mathrm{m}$ length is long enough for considering the laser heating and bending effect. The mesh is uniform in the $x$ and $y$ directions and the element sizes are increased by stretching in the $z$-directions. Eight-node 3D solid elements are applied in the analyses. The number of element in each of the fine regions is (in $x, y, z$ ): $60 \times 15 \times$ 8 . The total element number is 57,808 . Mesh tests are

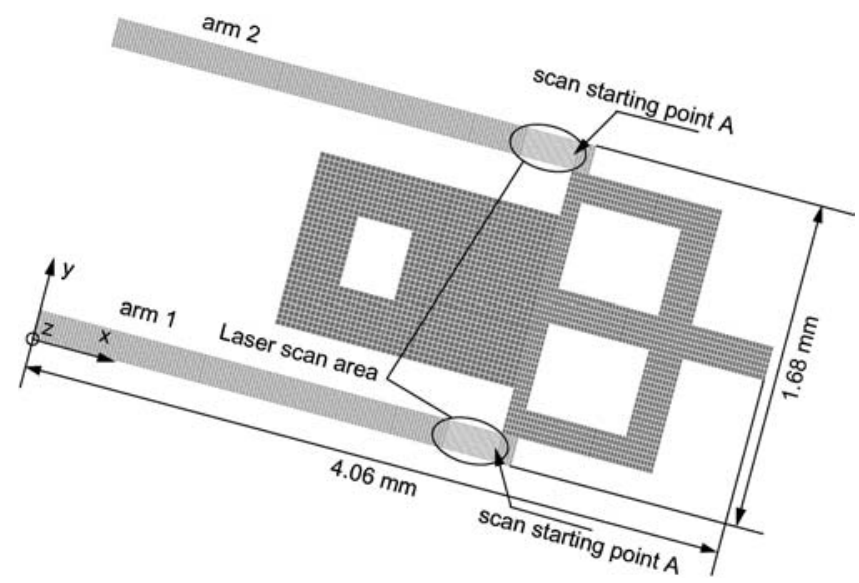

Fig. 2 Dimensions of the 3D model of a hard disk suspension 
Table 1 Parameters of $\mathrm{CW}$ laser

CW laser

Laser wavelength

Laser power

Laser beam diameter

Laser scan speed

$1.064 \mu \mathrm{m}$

$10-20 \mathrm{~W}$

$80 \mu \mathrm{m}$

$2 \mathrm{~m} / \mathrm{s}$

conducted by increasing the number of elements until the calculation result is independent of the mesh density.

The left ends of suspension arms are completely restrained and no other boundary restrain is applied. Two $\mathrm{CW}$ laser beams scan the arms of the suspension along the center line of each arm and in the negative $x$-direction, starting from point "A" as shown in Fig. 2. The scan lengths are the same if only the pitch of suspension needs to be adjusted. To adjust the roll of suspension, different scan lengths are applied onto the two arms.
The CW laser parameters used in the simulation are listed in Table 1. The non-linear finite element solver, ABAQUS is employed for the simulation.

\section{Results and discussion}

Figure 3 shows the temperature distribution at the moment $46 \mu \mathrm{s}$ from the beginning of laser scanning. Two identical laser beams scan along the negative $x$-direction as shown in Fig. 2. The power of each laser beam is $20 \mathrm{~W}$ and the scan speed is $2 \mathrm{~m} / \mathrm{s}$. Other laser parameters are as listed in Table 1. The peak temperature obtained is $1396 \mathrm{~K}$, lower than the melting point of stainless steel. Figure 3b shows the details of the heataffected zone on one of the arms. The center of the laser scan area has the highest temperature. The heat-affected zone in the $y$-direction is about $100 \mu \mathrm{m}$, which is slightly larger than the laser beam diameter, $80 \mu \mathrm{m}$. Figure 4 is
Fig. 3 Laser scanning induced temperature distribution at $46 \mu$ s (laser power $20 \mathrm{~W}$ ), a whole domain, b zoom-in of one suspension arm

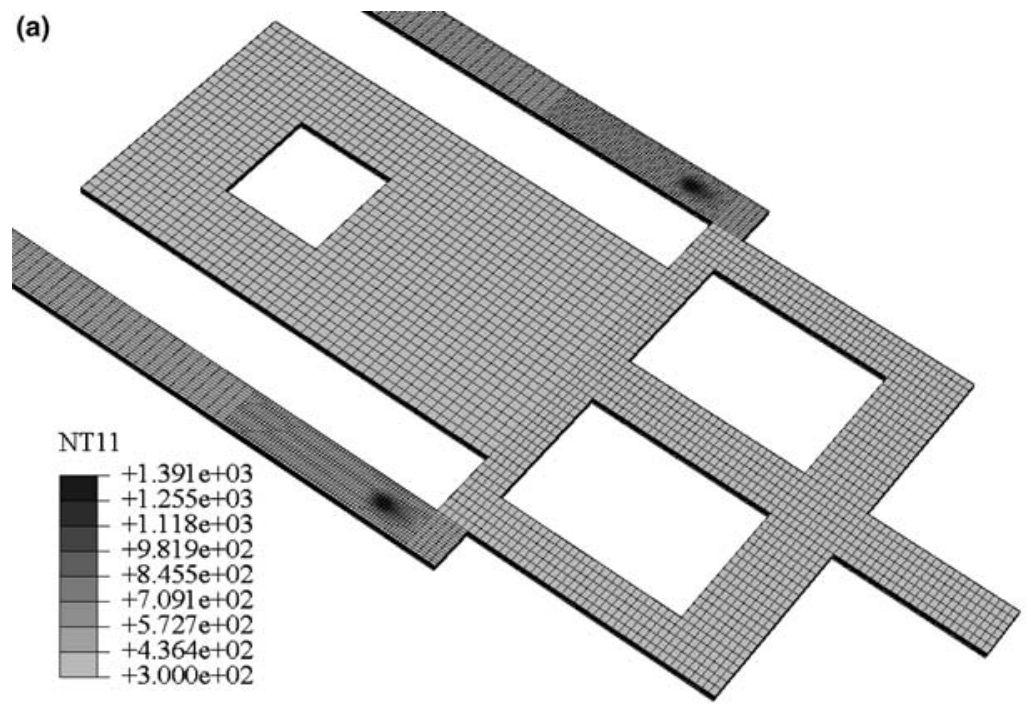

(b)

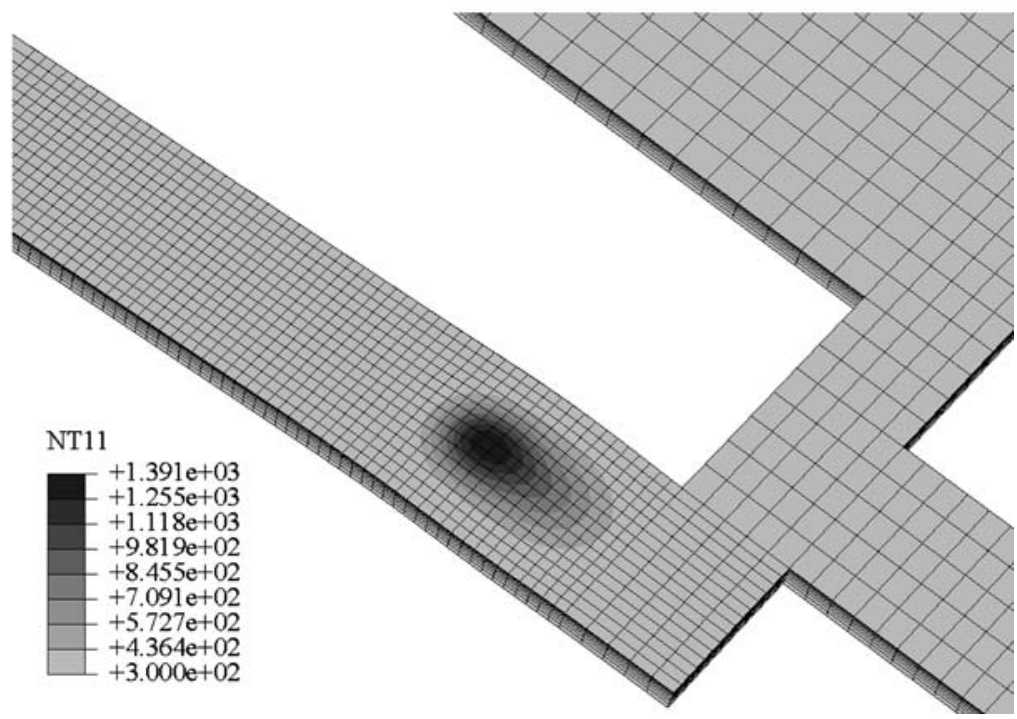




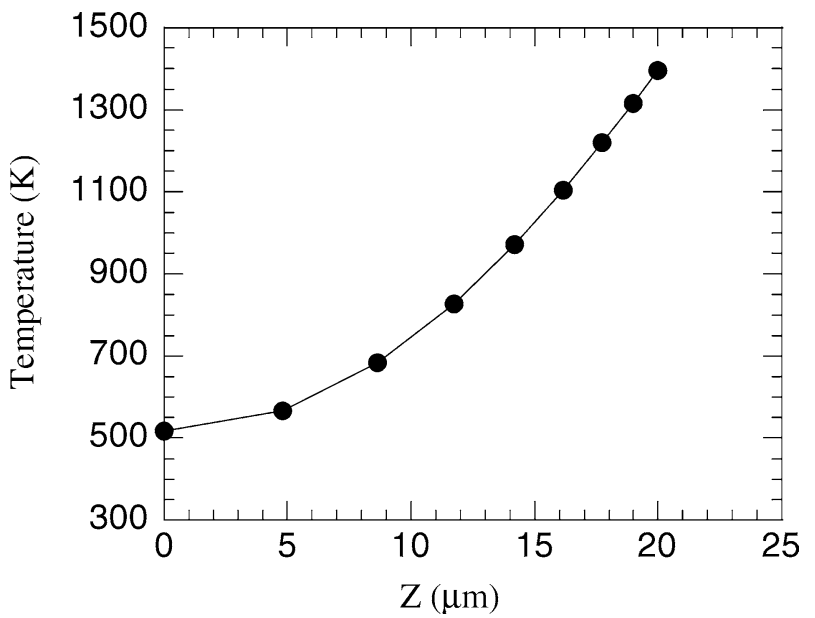

Fig. 4 Laser scanning induced temperature profile along the thickness direction at $46 \mu \mathrm{s}(x=2580 \mu \mathrm{m}, y=75 \mu \mathrm{m}$, laser power $20 \mathrm{~W}$ )

the temperature profile along the thickness direction (the $z$-direction) with the $x$ and $y$ position at 2580 and $75 \mu \mathrm{m}$, respectively. The temperature gradient within $10 \mu \mathrm{m}$ depth is about $70 \mathrm{~K} / \mu \mathrm{m}$. This sharp temperature gradient causes non-uniform plastic strains in the target and the permanent bending deformation after laser heating.

The residual plastic strain $\varepsilon_{x x}$ distribution, which is responsible for bending after the complete scanning of $100 \mu \mathrm{m}$ is shown in Fig. 5. As predicted by the temperature gradient mechanism, the compressive strain component along the $x$-direction is produced after the laser scanning. The compressive residual strain $\varepsilon_{x x}$ on the top surface implies that the suspension bends upward.

The permanent off-plane displacement $w$ (suspension deflection) after laser scanning is shown in Fig. 6. It can be seen that suspension bends in counter-clockwise (upward) direction after laser scanning. Due to the

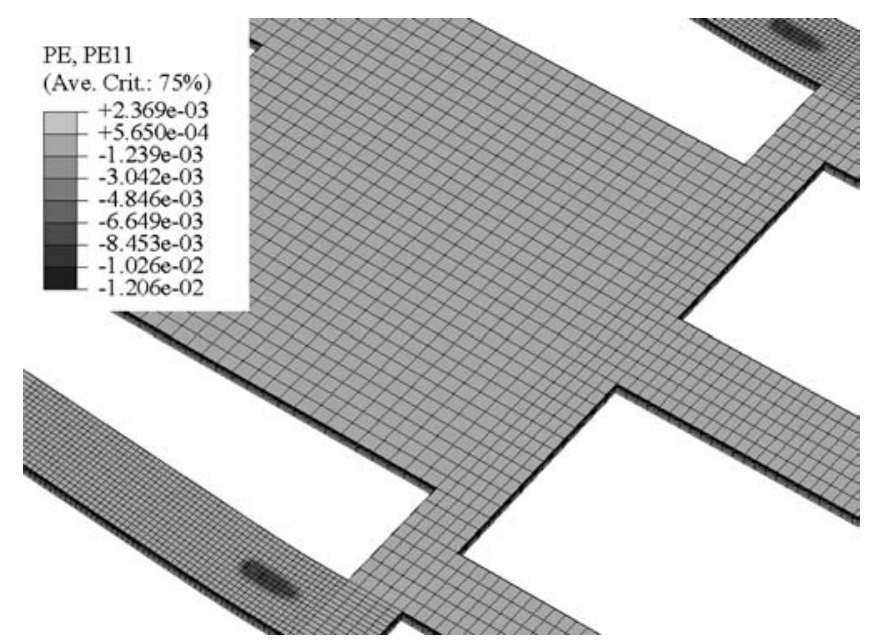

Fig. 5 Laser scanning induced residual plastic strain $\varepsilon_{x x}$ distribution (laser power $20 \mathrm{~W}$, total scan length $100 \mu \mathrm{m}$ ) identical laser scanning in both arms, only a pitch angle is produced. The deflection of the suspension tip is $11.94 \mu \mathrm{m}$, which is calculated to be $0.394^{\circ}$ in term of the pitch angle (The distance between the tip and the constrained position is $1.73 \mathrm{~mm}$ ).

Different imperfections, i.e., different combinations of pitch and roll, occur during suspension manufacturing. Therefore, different laser parameters are needed to correct the imperfections. In this work, the correlation between the bending angle (pitch) and the laser power is studied first. Five levels of laser power from 10 to $20 \mathrm{~W}$ are chosen and the scan length is fixed at $100 \mu \mathrm{m}$. The results are shown in Fig. 7. The solid dots are calculation results and the dashed line is the second order polynomial fitted curve, which can be expressed as:

$\alpha=0.264-0.06 \mathrm{P}+0.003 \mathrm{P}^{2}$

where $\alpha$ is the bending angle (degree) and $P$ is the laser power (W). According to Fig. 7, an appreciable bending is obtained when the laser power is higher than $10 \mathrm{~W}$ with the scan length of $100 \mu \mathrm{m}$. The reason that a laser power lower than $10 \mathrm{~W}$ does not generate bending is that the peak temperature achieved is not high enough to cause plastic deformation, which is responsible for permanent bending. To achieve larger bending angle, one can use higher laser power, and an angle as large as $0.4^{\circ}$ can be obtained with the laser power of $20 \mathrm{~W}$. The peak temperate achieved using $20 \mathrm{~W}$ laser power is $1396 \mathrm{~K}$. Using a laser power higher than $20 \mathrm{~W}$ while maintaining the same scanning speed will have the risk of damaging the suspension surface.

The correlation between the bending angle and the laser scan length is studied next. The laser power is fixed at $20 \mathrm{~W}$ and four scan lengths are used: 50,100, 150, and $200 \mu \mathrm{m}$. This laser power and scan length range are chosen because they can produce or correct the amount of pitch found in most suspensions imperfections. The results are shown in Fig. 8. The solid dots are calculating results and the dashed line is the linear fit, which can be expressed as:

$\alpha=-0.111+0.005 P$

The maximum bending angle obtained at the scan speed of $2 \mathrm{~m} / \mathrm{s}$ is $0.926^{\circ}$. The corresponding laser power is $20 \mathrm{~W}$ and the scan length is $200 \mu \mathrm{m}$. By interpolation between the scan length of 50 and $200 \mu \mathrm{m}$, the scan length required for adjusting a given pitch between 0.2 and $0.9^{\circ}$ can be found.

In summary of the above simulation results, it is found that the pitch angle without roll can be produced using identical laser scanning lengths in both suspension arms. Larger laser power and longer scan length will produce a larger bending angle.

The same procedure is used to calculate the roll angle by using different laser scan lengths in the two arms. It is necessary to point out that pitch is always produced along with roll during laser scanning, although pitch can be produced alone without roll. For an actual piece of 
Fig. 6 Laser scanning induced deformation distribution (laser power $20 \mathrm{~W}$, total scan length $100 \mu \mathrm{m}$ )

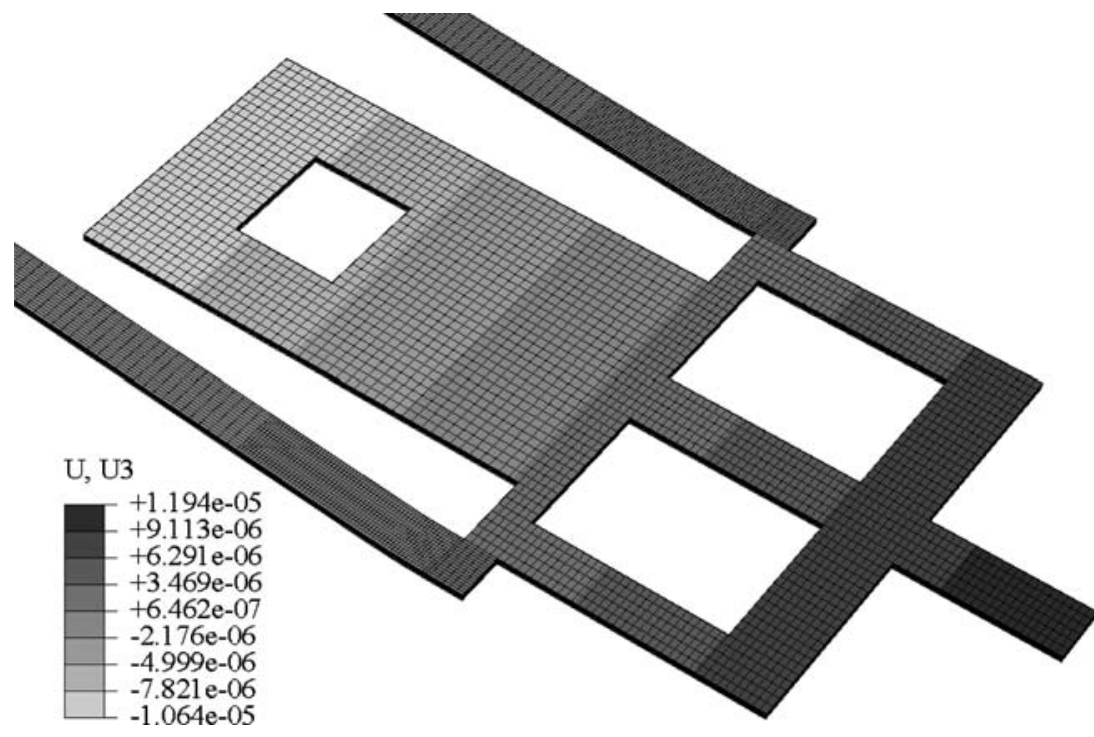

suspension, it has both pitch and roll, which are needed to be corrected by laser bending technique. Therefore, a data table will be useful if one wants to determine the

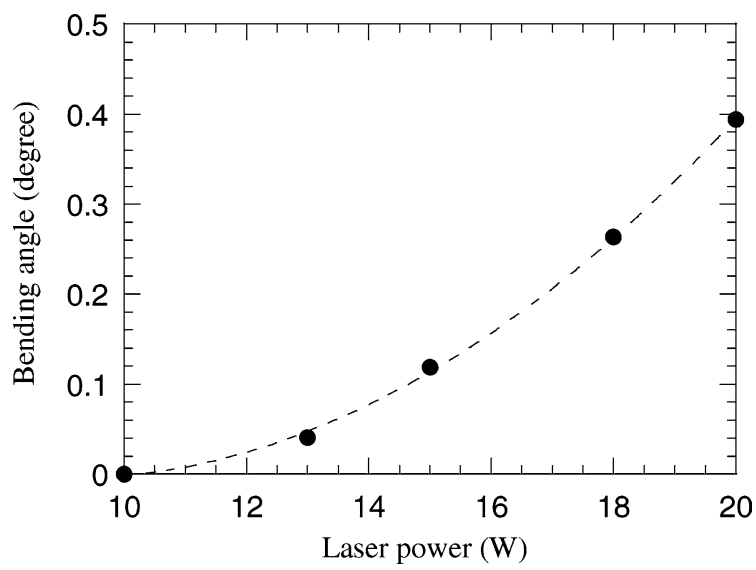

Fig. 7 Bending angle versus laser power with the same scan length $100 \mu \mathrm{m}$ (scanning speed $2 \mathrm{~m} / \mathrm{s}$ )

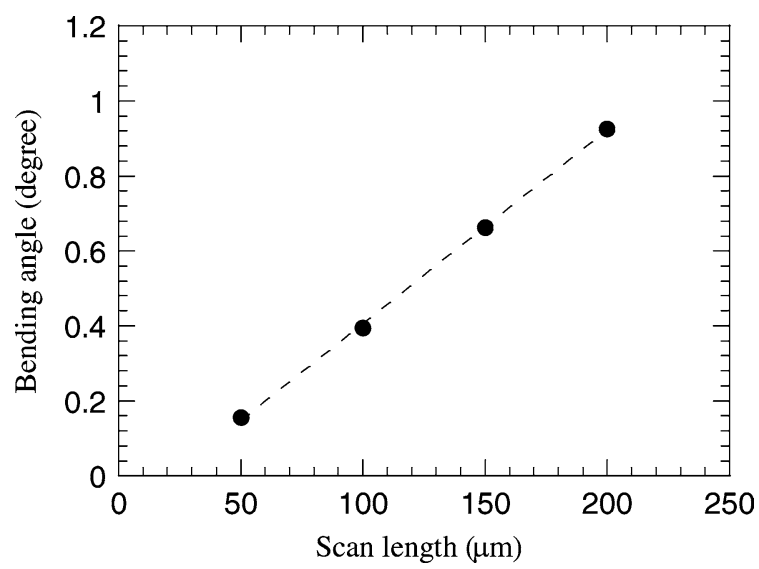

Fig. 8 Bending angle versus laser scan length with the same laser power $20 \mathrm{~W}$ (scanning speed $2 \mathrm{~m} / \mathrm{s}$ ) laser parameters for a given pitch and roll of the suspension. Extensive simulations are performed using the same laser power of $20 \mathrm{~W}$ but different scan lengths in the two arms. Again, this laser power is used since it can adjust the most pitch/roll combinations found in a suspension. Table 2 summarizes the resulting pitch and roll for each case. The pitch angle toward laser beam is defined as positive sign. The sign of the roll angle are determined by the right hand rule.

As shown in Table 2, a pair of symmetric (identical laser parameters and scan lengths) scans always gives a pitch angle only whereas a pair of scans with different lengths gives pitch and roll angles simultaneously. The largest pitch can be induced is $0.926^{\circ}$ and the largest roll is $0.11^{\circ}$. According to this table, data interpolation can be performed and a matrix of pitch and roll produced by laser scanning can be established. Contours of pitch and roll angles are plotted after the data interpolation as shown in Fig. 9. For a given set of pitch/roll, one needs to first find the two curves corresponding to the pitch and roll, and then find the interception point of these two curves to determine the two scan lengths. These two scan lengths are the predicted laser scan lengths for correcting the given pitch/roll.

The effects of uncertainties are also studied. Sample thickness and material yield strength are the two of the most common uncertainties in a suspension. In this work, we study the deviation with the difference in thickness of $\pm 1.5 \mu \mathrm{m}$ and the yield strength of $\pm 10 \%$. The simulation results are summarized in Table 3. The same laser power of $20 \mathrm{~W}$ is used. Four different scan lengths with different thicknesses or yield strengths are calculated. According to this table, the pitch and roll increase when the thickness and yield stress decrease and the pitch and roll decrease when the thickness and yield stress increase. From bending theory it is known that the bending angle is approximately inversely proportional to the square of the specimen thickness. From the calculation, it is found that if the thickness is reduced from 20 
Table 2 Pitch (the first value) and roll (the second value) angles produced by different laser scan lengths at a laser power of $20 \mathrm{~W}$ (unit: degree)

\begin{tabular}{llllll}
\hline Scan lengths $(\mu \mathrm{m})$ & $(\operatorname{arm} 2) 0$ & 50 & 100 & 150 & 200 \\
\hline$(\operatorname{arm~1)} 0$ & & $0.078: 0.023$ & $0.208: 0.055$ & $0.339: 0.084$ & $0.467: 0.110$ \\
50 & $0.078:-0.023$ & $0.155: 0.000$ & $0.286: 0.033$ & $0.413: 0.060$ & $0.544: 0.087$ \\
100 & $0.208:-0.055$ & $0.286:-0.033$ & $0.394: 0.000$ & $0.534: 0.031$ & $0.668: 0.058$ \\
150 & $0.339:-0.084$ & $0.413:-0.060$ & $0.534:-0.031$ & $0.663: 0.000$ & $0.769: 0.024$ \\
200 & $0.467:-0.110$ & $0.544:-0.087$ & $0.668:-0.058$ & $0.769:-0.024$ & $0.926: 0.000$ \\
\hline
\end{tabular}

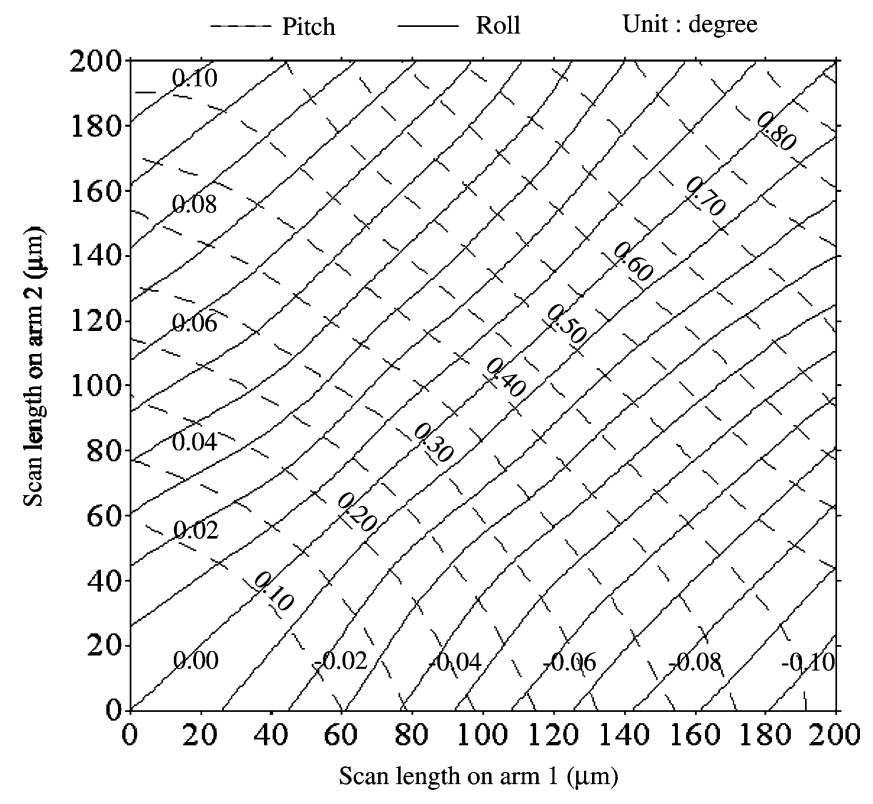

Fig. 9 Contours of pitch (dash line) and roll (solid line) obtained with different scan lengths (laser power $20 \mathrm{~W}$ )

to $18.5 \mu \mathrm{m}$, which is $92.5 \%$ of the original value $(20 \mu \mathrm{m})$, the bending angle is increased by about $17 \%$, which agrees with the theory. The effect of the yield stress can be understood as that a larger plastic strain will be produced with a lower material yield stress under the identical load condition, and consequently a larger bending angle will be produced.

\section{Conclusions}

A 3D FEA model is employed to calculate laser bending of hard drive suspensions and extensive numerical calculations have been carried out. It is shown that bending resolution as high as $0.01^{\circ}$ can be achieved.
With the laser power of $20 \mathrm{~W}$ and the scan length varying from 50 to $200 \mu \mathrm{m}$, the largest pitch/roll angles achieved in the laser bending are $0.926 / 0.110^{\circ}$. A larger range of pitch and roll angles can be obtained if a longer scan length is used. By interpolation between these results, a matrix of pitch/rollversus scan length is obtained. The 2D contours of pitch/roll are plotted according to the calculated matrix, which can be used to predict laser scan lengths for correcting the given pitch/ roll imperfection. The effect of uncertainties, such as the sample thickness and the material yield strength, are studied and it is found that a smaller thickness and yield stress increases the pitch and roll.

\section{References}

Bogy DB, Zeng QH (1999) Effects of suspension limiters on the dynamic load/unload process: numerical simulation. IEEE Trans Magn 35(5):2490-2492

Chen G, Xu X, Poon CC, Tam AC (1998) Laser-assisted microscale deformation of stainless steels and ceramics. Opt Eng 37(10):2837-2842

Chen G, Xu X, Poon CC, Tam AC (1999) Experimental and numerical studies on microscale bending of stainless steel with pulsed laser. ASME J Appl Mech 66(5):772-779

Frank T, Neidhart W, Talke FE (2000) Optimization of a hard disk drive using finite element analysis. Invited paper, Special Publication of the ASME/STLE proceedings

Geiger M, Vollertsen F (1993) The mechanism of laser forming. Ann CIRP 42:301-304

Kilian S, Zander U, Talke FE (2003) Suspension modeling and optimization using finite element analysis. Tribol Int 36(4):317324

Maykuth DJ (1980) Structural alloys handbook, vol 2: metals and ceramics information center. Battelee Columbus Laboratories, Columbus, OH, pp1-61

Singh GP, Wu X, Brown BR, Kozlovsky W (2001) Laser gram load adjust for improved disk drive performance. IEEE Trans Magn 37(2):959-963

Takahashi H, Bogy DB, Matsumoto M (1998) Vibration of head suspensions for proximity recording. IEEE Trans Magn 34(4):1756-1758

Table 3 Comparison of pitch and roll produced with different thickness and yield stress during laser bending (unit: degree, laser power: $20 \mathrm{~W})$

\begin{tabular}{llllll}
\hline $\begin{array}{l}\text { Scan lengths } \\
(\mu \mathrm{m})\end{array}$ & Original pitch/roll & Thickness $18.5 \mu \mathrm{m}$ & Thickness $21.5 \mu \mathrm{m}$ & Yield stress $-10 \%$ & Yield stress $+10 \%$ \\
\hline $200 / 50$ & $0.544 / 0.087$ & $0.626: 0.099+15 /+14 \%$ & $0.481 / 0.077-12 /-12 \%$ & $0.601 / 0.095+10 /+9 \%$ & $0.494 / 0.081-9 /-7 \%$ \\
$200 / 100$ & $0.668 / 0.058$ & $0.778 / 0.062+16 /+7 \%$ & $0.592 / 0.049-11 /-15 \%$ & $0.734 / 0.062+10 /+7 \%$ & $0.605 / 0.049-9 /-15 \%$ \\
$200 / 150$ & $0.769 / 0.024$ & $0.924 / 0.030+20 /+25 \%$ & $0.705 / 0.022-8 /-8 \%$ & $0.891 / 0.029+16 /+21 \%$ & $0.725 / 0.023-6 /-4 \%$ \\
$200 / 200$ & $0.926 / 0.000$ & $1.072 / 0.000+16 / 0 \%$ & $0.888 / 0.000-4 / 0 \%$ & $1.032 / 0.000+11 / 0 \%$ & $0.840 / 0.000-9 / 0 \%$ \\
\hline
\end{tabular}


Takeuti Y, Komori S, Noda N, Nyuko H (1979) Thermal-stress problems in industry 3: temperature dependency of elastic moduli for several metals at temperatures from -196 to $1000^{\circ} \mathrm{C}$. J Therm Stresses 2:233-250

Wilson C, Bogy DB (1994) Model analysis of suspension system. ASME J Eng Ind 116(2):377-386

Weissner S, Zander U, Talke FE (2003) A new finite-element based suspension model including displacement limiters for load/unload simulation. ASME J Tribol 125(1):162-167
Zhang XR, Xu X (2003) High precision microscale bending by pulsed and CW lasers. ASME J Manuf Sci Eng 125(3):512518

Zhang XR, Chen G, Xu X (2002) Numerical simulation of pulsed laser bending. ASME J Appl Mech 69(3):254-260 